\title{
Screening masses in the SU(3) pure gauge theory and universality
}

\author{
R. Falcone ${ }^{a *}$, R. Fiore ${ }^{a}$, M. Gravina ${ }^{a}$ and A. Papa ${ }^{a}$ \\ ${ }^{a}$ Dipartimento di Fisica, Università della Calabria, \\ and Istituto Nazionale di Fisica Nucleare, Gruppo collegato di Cosenza \\ I-87036 Arcavacata di Rende, Cosenza, Italy \\ E-mail: rfalcone, fiore, gravina, papaecs.infn.it
}

\begin{abstract}
We determine from Polyakov loop correlators the screening masses in the deconfined phase of the $(3+1) d \mathrm{SU}(3)$ pure gauge theory at finite temperature near the transition, for two different channels of angular momentum and parity. Their ratio is compared with that of the massive excitations with the same quantum numbers in the $3 d$ 3-state Potts model in the broken phase near the transition point at zero magnetic field. Moreover we study the inverse decay length of the correlation between the real parts and between the imaginary parts of the Polyakov loop and compare the results with expectations from perturbation theory and mean-field Polyakov loop models.
\end{abstract}

The XXV International Symposium on Lattice Field Theory

July 30-4 August 2007

Regensburg, Germany

${ }^{*}$ Speaker. 


\section{Introduction}

In this work we compare the spectrum of the inverse decay lengths of Polyakov loop correlators in the $(3+1) d \mathrm{SU}(3)$ gauge theory in the deconfined phase near the transition with the spectrum of massive excitations of the $3 d$ 3-state Potts model in the broken phase near the transition at zero magnetic field, which were determined in Ref. [1]. The aim of the work is to verify if and to what extent the Svetitsky-Yaffe conjecture [2] also holds for theories which undergo a weakly first order phase transition, using mass ratios as a probe. In particular we focus on the low-lying masses in two different sectors of parity and orbital angular momentum, $0^{+}$and $2^{+}$. We expect that, if universality would apply in strict sense, these spectra should exhibit the same pattern, as suggested by several numerical determinations in the $3 d$ Ising class $[3,4,5,6]$.

We extend our numerical analysis to temperatures far away from the transition temperature $T_{t}$ in order to look for possible "scaling" of the fundamental masses with temperature. Moreover, we consider also the screening masses resulting from correlators of the real parts and of the imaginary parts of the Polyakov loop. These determinations can represent useful benchmarks for effective models of the high-temperature phase of SU(3), such as those based on mean-field theories of the Polyakov loop, suggested by R. Pisarski [7].

\section{Screening masses from Polyakov loop correlators}

Screening masses are defined as the inverse decay lengths of the Yukawa-like potential between two static sources. They are generally determined through the correlation of suitable operators. In our case correlations are between operators with different spatial separation. The general large distance behavior for the correlation function $G\left(\left|z_{1}-z_{2}\right|\right)$, in an infinite lattice, is:

$$
G\left(\left|z_{1}-z_{2}\right|\right)=\sum_{n} a_{n} e^{-m_{n}\left|z_{1}-z_{2}\right|}
$$

where $m_{0}$ is the fundamental mass, while $m_{1}, m_{2}, \ldots$ are higher masses with the same angular momentum and parity quantum numbers of the fundamental mass. On a periodic lattice the above equation must be modified by the inclusion of the so called "echo" term:

$$
G\left(\left|z_{1}-z_{2}\right|\right)=\sum_{n} a_{n}\left[e^{-m_{n}\left|z_{1}-z_{2}\right|}+e^{-m_{n}\left(N_{z}-\left|z_{1}-z_{2}\right|\right)}\right] .
$$

The fundamental mass in a definite channel can be extracted from wall-wall correlators by looking for a plateau of the effective mass at large distances,

$$
m_{\mathrm{eff}}(z)=\ln \frac{G(z-1)}{G(z)} .
$$

In the $0^{+}$-channel, the connected wall-wall correlator in the $z$-direction is defined as

$$
G\left(\left|z_{1}-z_{2}\right|\right)=\operatorname{Re}\left\langle\bar{P}\left(z_{1}\right) \bar{P}\left(z_{2}\right)^{\dagger}\right\rangle-|\langle P\rangle|^{2},
$$

where

$$
\bar{P}(z)=\frac{1}{N_{x} N_{y}} \sum_{n_{x}=1}^{N_{x}} \sum_{n_{y}=1}^{N_{y}} P\left(n_{x} a, n_{y} a, z\right),
$$


represents the Polyakov loop averaged over the $x y$-plane at a given $z .{ }^{1}$ The wall average implies the projection at zero momentum in the $x y$-plane.

For the $2^{+}$-channel, we used the variational method $[8,9]$ (for more details, see [10] and references therein.)

Our choice of wall-averaged operators in the $2^{+}$-channel is inspired by Ref. [11] and reads

$$
\bar{P}_{n}(z)=\frac{1}{N_{x} N_{y}} \sum_{n_{x}=1}^{N_{x}} \sum_{n_{y}=1}^{N_{y}} P\left(n_{x} a, n_{y} a, z\right)\left[P\left(n_{x} a+n a, n_{y} a, z\right)-P\left(n_{x} a, n_{y} a+n a, z\right)\right] .
$$

In most cases we have taken 8 operators, corresponding to different values of $n$, with the largest $n$ almost reaching the spatial lattice size $N_{x}$.

We consider also correlators of the (wall-averaged) real and imaginary parts of the Polyakov loop, defined as

$$
\begin{aligned}
G_{R}\left(\left|z_{1}-z_{2}\right|\right) & =\left\langle\operatorname{Re} \bar{P}\left(z_{1}\right) \operatorname{Re} \bar{P}\left(z_{2}\right)\right\rangle-\left\langle\operatorname{Re} \bar{P}\left(z_{1}\right)\right\rangle\left\langle\operatorname{Re} \bar{P}\left(z_{2}\right)\right\rangle \\
G_{I}\left(\left|z_{1}-z_{2}\right|\right) & =\left\langle\operatorname{Im} \bar{P}\left(z_{1}\right) \operatorname{Im} \bar{P}\left(z_{2}\right)\right\rangle .
\end{aligned}
$$

The corresponding screening masses, $\hat{m}_{R}$ and $\hat{m}_{I}$, can be extracted in the same way as for the $0^{+}$mass. We have studied the ratio $m_{I} / m_{R}$ over a wide interval of temperatures above the transition temperature $T_{t}$ of (3+1)d SU(3) and seen how it compares with the prediction from hightemperature perturbation theory, according to which it should be equal to $3 / 2$ [12, 13], and with the prediction from the mean-field Polyakov loop model of Ref. [14], according to which it should be equal to 3 in the transition region. The interplay between the two regimes should delimit the region where mean-field Polyakov loop models should be effective.

\section{Numerical results}

We used the Wilson lattice action and generated Monte Carlo configurations by a combination of the modified Metropolis algorithm [15] with over-relaxation on SU(2) subgroups [16]. The error analysis was performed by the jackknife method over bins at different blocking levels. We performed our simulations on a $16^{3} \times 4$ lattice, for which $\beta_{t}=5.6908$ (2) [17], over an interval of $\beta$ values ranging from 5.69 to 9.0 .

Screening masses are determined from the plateau of $m_{\mathrm{eff}}(z)$ as a function of the wall separation $z$. In each case, the plateau mass is taken as the effective mass (with its error) belonging to the plateau and having the minimal uncertainty. We define plateau the largest set of consecutive data points, consistent with each other within $1 \sigma$. This procedure is more conservative than identifying the plateau mass and its error as the results of a fit with a constant on the effective masses $m_{\text {eff }}(z)$, for large enough $z$.

Just above the critical value $\beta_{t}$ we find a large correlation length, which is not of physical relevance. It is instead a genuine finite size effect [18] related to tunneling between degenerate vacua. This effect disappears by going to larger lattice volumes or moving away from $\beta_{t}$ in the deconfined phase. Tunneling can occur between the symmetric and the broken phase, and between

\footnotetext{
${ }^{1}$ Here and in the following, $N_{i}(i=x, y, z)$ is the number of lattice sites in the $i$-direction.
} 

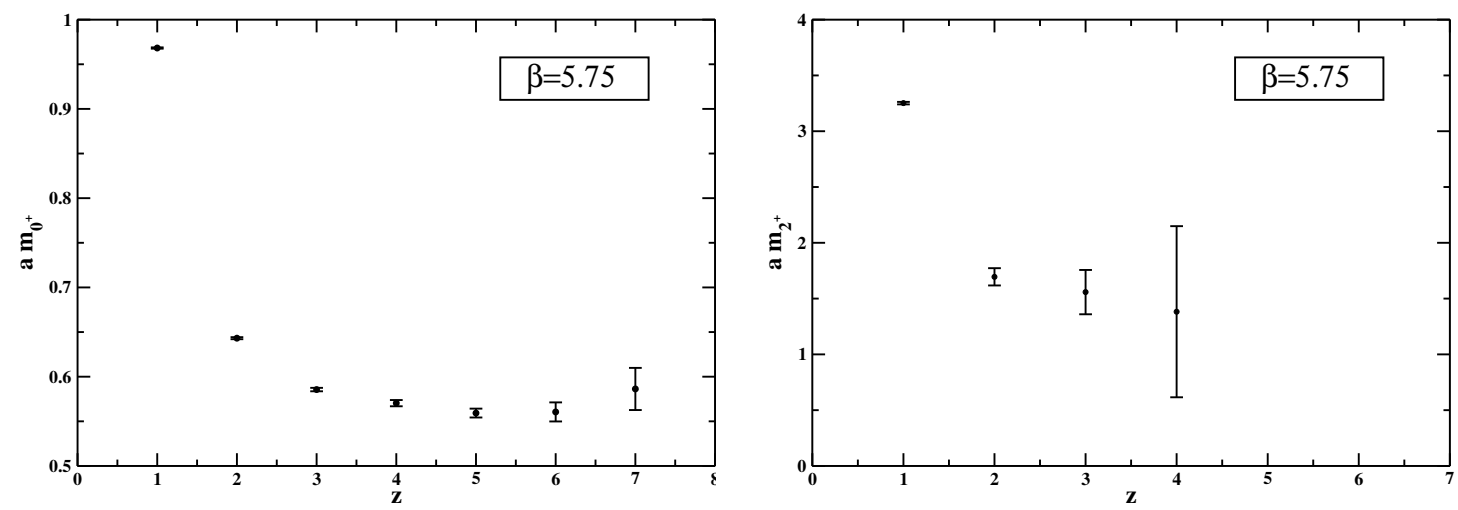

Figure 1: Effective mass in the $0^{+}$(left) and the $2^{+}$(right) channel as a function of the separation between walls on the $(x, y)$ plane at $\beta=5.75$.

the three degenerate vacua of the deconfined phase. When tunneling is active, the correlation function has the following expression [18]:

$$
G\left(\left|z_{1}-z_{2}\right|\right) \sim a_{0} e^{-m_{0}\left|z_{1}-z_{2}\right|}+b_{0} e^{-m_{t}\left|z_{1}-z_{2}\right|},
$$

where $m_{t}$ is the inverse of the tunneling correlation length and is generally much smaller than the fundamental mass $m_{0}$ and therefore behaves as a constant additive term in the correlation function. ${ }^{2}$ The dependence on $m_{t}$ in the correlation function can be removed by extracting the effective mass by use of the combination

$$
m_{\mathrm{eff}}(z)=\ln \frac{G(z)-G(z+1)}{G(z+1)-G(z+2)} .
$$

A typical example of the behavior of the effective mass with $z$ is shown in Fig. 1 for the $0^{+}$and the $2^{+}$channels. In Figs. 2 and 3 we show the behavior with $\beta$ of $\hat{m}_{0^{+}}, \hat{m}_{2^{+}}, \hat{m}_{R}$ and $\hat{m}_{I}$.

We observe from that $\hat{m}_{0^{+}}$and $\hat{m}_{R}$ are consistent within statistical errors, this indicating that the Polyakov loop correlation is dominated by the correlation between the real parts. We can see that the fundamental mass in the $0^{+}$channel, as well as $\hat{m}_{R}$, becomes much smaller than 1 at $\beta_{t}$, as expected for a weakly first order phase transition. In the cases of $\hat{m}_{0^{+}}$and of $\hat{m}_{R}$ we have made some determinations below $\beta_{t}$ (see Figs. 2 and 3). It turns out that masses in lattice units take their minimum value just at $\beta_{t}$, where there is a "cusp" in the $\beta$-dependence. Such a behaviour was observed also by the authors of Ref. [19], whose results, when the comparison is possible, agree with ours. We have also looked for a scaling law for the fundamental mass in the $0^{+}$channel, but with the understanding that any second-order-like scaling law, when applied to the region near a first order phase transition, should be taken as an effective description, which cannot hold too close to the transition point. With this spirit, we have compared our data with the scaling law

$$
\left(\frac{\beta_{1}-\beta_{t}}{\beta_{2}-\beta_{t}}\right)^{v} \sim \frac{\hat{m}_{0^{+}}\left(\beta_{1}\right)}{\hat{m}_{0^{+}}\left(\beta_{2}\right)},
$$

\footnotetext{
${ }^{2}$ In (3.1) we have taken into account only the lowest masses in the spectrum and, for brevity, omitted to write the "echo" terms.
} 

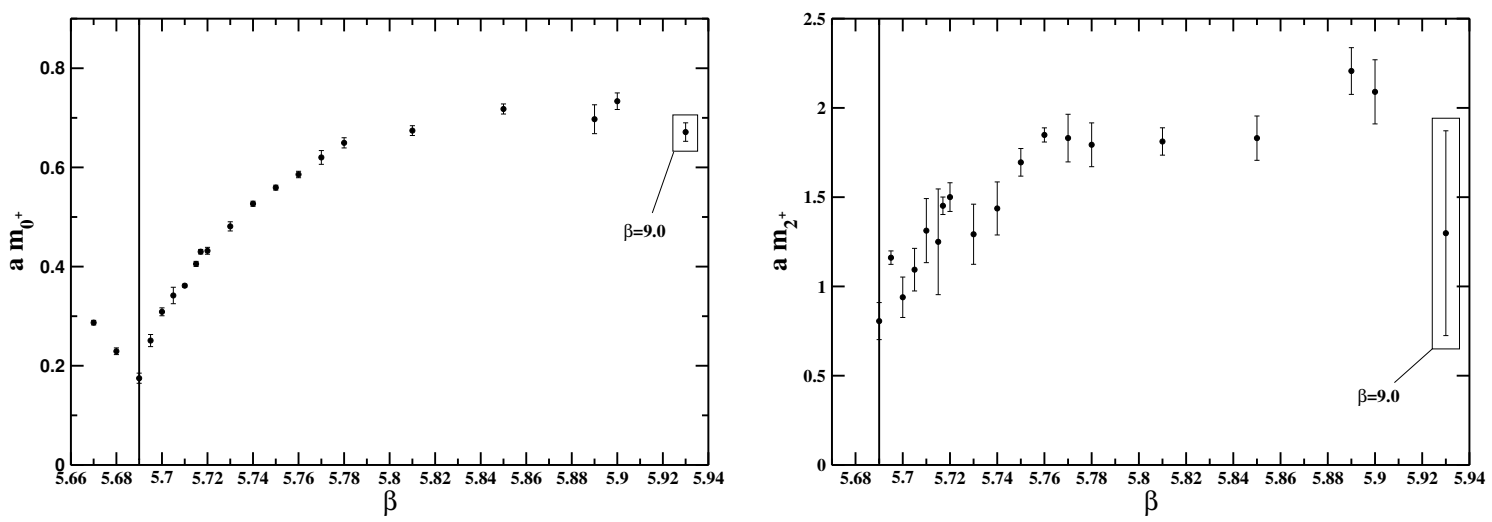

Figure 2: Screening mass in the $0^{+}$channel (left) and in the $2^{+}$channel (right) vs. $\beta$.
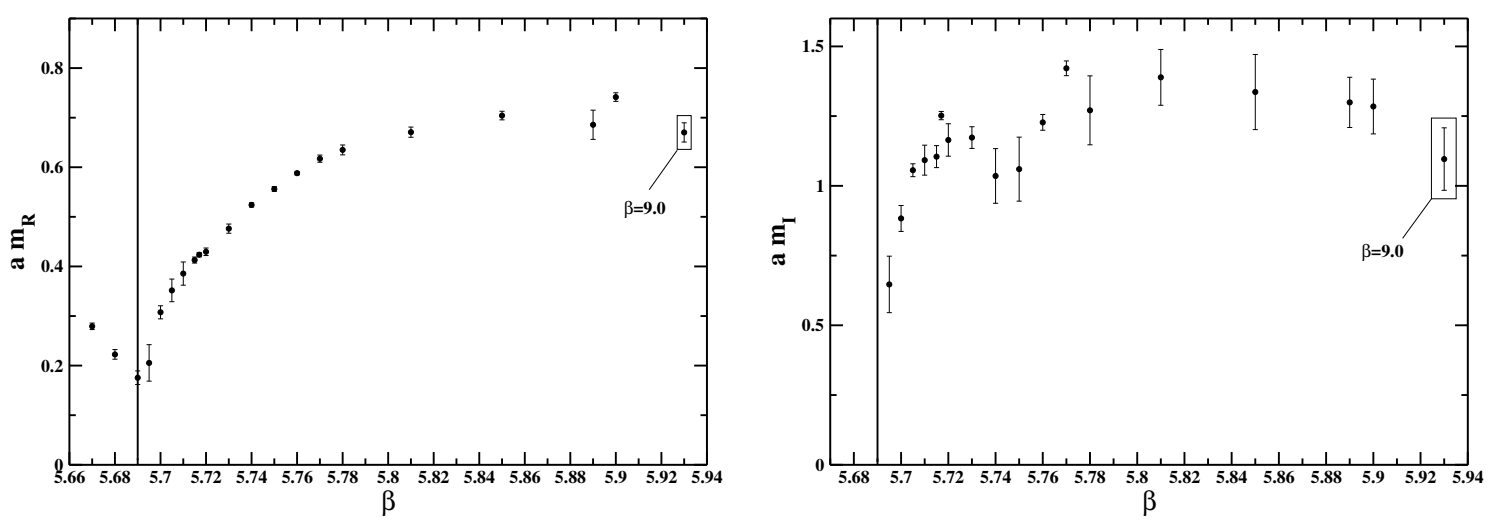

Figure 3: Screening masses $\hat{m}_{R}$ (left) and $\hat{m}_{I}$ (right) vs. $\beta$.

where $\hat{m}_{0^{+}}\left(\beta_{1}\right)$ and $\hat{m}_{0^{+}}\left(\beta_{2}\right)$ are the fundamental masses in the $0^{+}$channel at $\beta_{1}$ and $\beta_{2}$, respectively. We have considered several choices of $\beta_{1}$ and found that for each of them there is a wide "window" of $\beta$ values above $\beta_{t}$ where the scaling law (3.3) works, with a "dynamical" exponent $v$ (see Ref. [10] for a details). For $\beta_{1}=5.72$ we have calculated also the $\chi^{2} /$ d.o.f. when $v$ is put exactly equal to $1 / 3$ (suggested in Ref. [20] to apply to the standard correlation function), getting $\chi^{2} /$ d.o.f. $=0.75$ in the window from $\beta=5.715$ to $\beta=5.78$. In Fig. 4 we show, for this choice of $\beta_{1}$, the comparison between data and the "scaling" function with $v$ set equal to $1 / 3$.

Then, we have considered the $\beta$-dependence of the ratio $m_{2^{+}} / m_{0^{+}}$, shown in Fig. 5 . We have found that this ratio can be interpolated with a constant in the interval from $\beta_{t}$ to $\beta=5.77$. This constant turned out to be 3.172(65), with a $\chi^{2} /$ d.o.f equal to 1.085 . In the fit we excluded the point at $\beta=5.695$, for which the determination of $m_{2^{+}}$is probably to be rejected. If the point at $\beta=5.695$ is included, the constant becomes 3.214(64) with $\chi^{2} /$ d.o.f $=2.21$. The fact that the ratio $m_{2^{+}} / m_{0^{+}}$ is compatible with a constant in the mentioned interval suggests that $\hat{m}_{2^{+}}$scales similarly to $\hat{m}_{0^{+}}$ near the transition. This constant turns out to be larger than the ratio between the lowest massive excitations in the same channels in the broken phase of the $3 d 3$-state Potts model, which was 


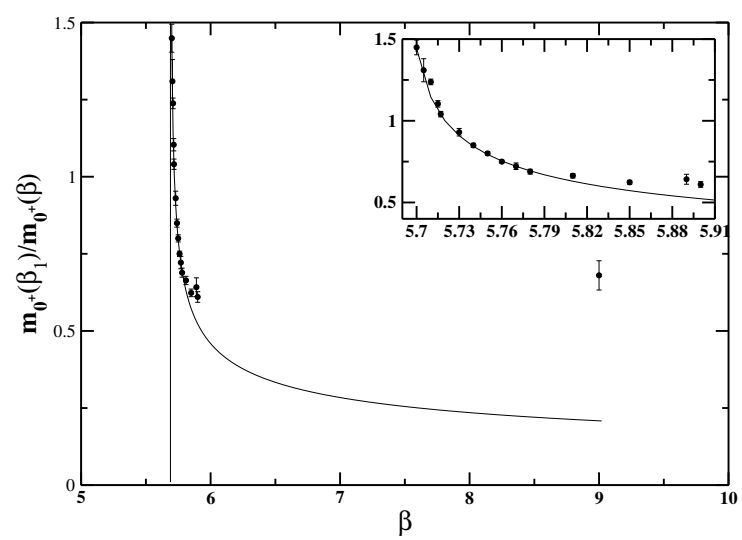

Figure 4: Comparison between the scaling function $\left[\left(\beta_{1}-\beta_{t}\right) /\left(\beta-\beta_{t}\right)\right]^{1 / 3}$ and the mass ratio $m_{0^{+}}\left(\beta_{1}\right) / m_{0^{+}}(\beta)$ for varying $\beta$, with $\beta_{1}=5.72$.
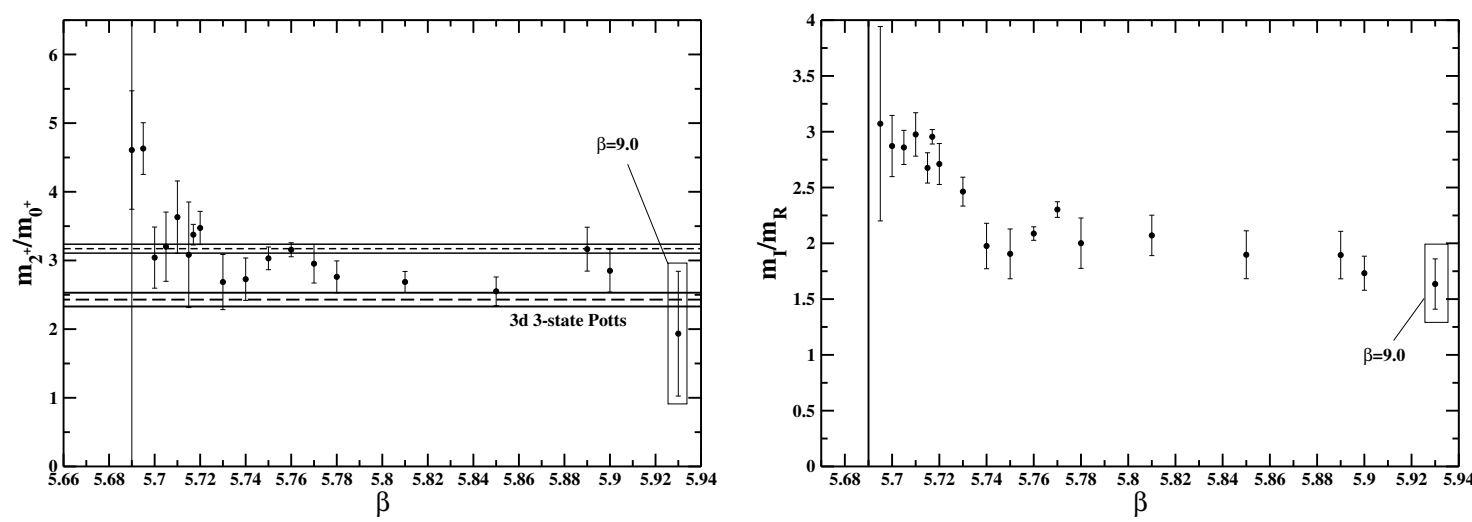

Figure 5: (Left) Ratio $m_{2^{+}} / m_{0^{+}}$as a function of $\beta$ in the deconfined phase. The three upper horizontal lines represent the constant (with its error) which fits the data (see the text for details); the three lower horizontal lines represent the corresponding mass ratio (with its error) found in the $3 d$ 3-state Potts model [1]. (Right) Ratio $m_{I} / m_{R}$ as a function of $\beta$ in the deconfined phase. The vertical line corresponds to the critical $\beta$ value.

determined in Ref. [1] to be 2.43(10).

We have calculated the ratio $m_{I} / m_{R}$ for $\beta$ ranging from 5.695 up to 9.0. We observe from the right panel of Fig. 5 that this ratio is compatible with $3 / 2$ at the largest $\beta$ values considered, in agreement with the high-temperature perturbation theory. Then, when the temperature is lowered towards the transition, this ratio goes up to a value compatible with 3 , in agreement with the Polyakov loop model of Ref. [14], which contains only quadratic, cubic and quartic powers of the Polyakov loop, i.e. the minimum number of terms required in order to be compatible with a first order phase transition. The same trend has been observed also in Ref. [19].

\section{Conclusions and outlook}

In this work we have studied in the $(3+1) d \mathrm{SU}(3)$ pure gauge theory above the deconfinement transition the lowest masses in the $0^{+}$and the $2^{+}$channels of angular momentum and parity and the 
screening masses resulting from the correlation between the real parts and between the imaginary parts of the Polyakov loop. The behavior of the ratio between the masses in the $0^{+}$and the $2^{+}$ channels with the temperature suggests that they have a common scaling above the transition temperature. This ratio turns to be $\simeq 30 \%$ larger than the ratio of the lowest massive excitations in the same channels of the $3 d$ 3-state Potts model in the broken phase. This can be taken as an estimate of the level of approximation by which the Svetitsky-Yaffe conjecture, valid in strict sense only for continuous phase transitions, can play some role also for $(3+1) d \mathrm{SU}(3)$ at finite temperature.

The dependence on the temperature of the ratio between the screening masses from the correlation between the real parts and between the imaginary parts of the Polyakov loop shows a nice interplay between the high-temperature regime, where perturbation theory should work, and the transition regime, where mean-field effective Polyakov loop models could apply.

\section{References}

[1] R. Falcone, R. Fiore, M. Gravina and A. Papa, Nucl. Phys. B 767 (2007) 385 [hep-lat/0612016].

[2] B. Svetitsky and L.G. Yaffe, Nucl. Phys. B 210 (1982) 423.

[3] M. Caselle, M. Hasenbusch and P. Provero, Nucl. Phys. B 556 (1999) 575 [hep-lat / 9903011 ].

[4] M. Caselle, M. Hasenbusch, P. Provero and K. Zarembo, Nucl. Phys. B 623 (2002) 474 [hep-th/0103130].

[5] R. Fiore, A. Papa and P. Provero, Nucl. Phys. (Proc. Suppl.) 119 (2003) 490 [hep-lat / 0208020 ].

[6] R. Fiore, A. Papa and P. Provero, Phys. Rev. D 67 (2003) 114508 [hep-lat/ 020802 0].

[7] R.D. Pisarski, Phys. Rev. D 62 (2000) 111501 [hep-ph / 0101168$].$

[8] A.S. Kronfeld, Nucl. Phys. (Proc. Suppl.) 17 (1990) 313.

[9] M. Lüscher and U. Wolff, Nucl. Phys. B 339 (1990) 222.

[10] R. Falcone, R. Fiore, M. Gravina and A. Papa, Nucl. Phys. B. 785 (2007) 19, [arXiv : 0704.3882 [hep-lat]].

[11] V. Agostini, G. Carlino, M. Caselle and M. Hasenbusch, Nucl. Phys. B 484 (1997) 331 [hep-lat/9607029].

[12] S. Nadkarni, Phys. Rev. D 33 (1986) 3738.

[13] A. Dumitru and R.D. Pisarski, Phys. Rev. D 66 (2002) 096003 [hep-ph / 0204223 ].

[14] A. Dumitru and R.D. Pisarski, Nucl. Phys. (Proc. Suppl.) 106 (2002) 483 [hep-ph/ 0110214 ].

[15] N. Cabibbo and E. Marinari, Phys. Lett. B 119 (1982) 387.

[16] S.L. Adler, Phys. Rev. D 23 (1981) 2901.

[17] G. Boyd, J. Engels, F. Karsch, E. Laermann, C. Legeland, M. Lutgemeier and B. Petersson, Nucl. Phys. B 469 (1996) 419 [hep-lat/ 9602007 ].

[18] R.V. Gavai, F. Karsch and B. Petersson, Nucl. Phys. B 322 (1989) 738 [cern-th-5221/88].

[19] S. Datta and S. Gupta, Phys. Rev. D 67 (2003) 054503 [hep-lat / 0208001 ].

[20] M.E. Fisher and A.N. Berker, Phys. Rev. B 26 (1982) 2507. 